\title{
First-in-human assays: excitement and introspection
}

\author{
"...human assay development and bioanalysis of clinical samples is considered to be the key milestone of \\ achievement for many budding scientists in the world of bioanalysis."
}

Is it not exciting to develop and validate new methods for novel chemical entities (NCEs) to support first-in-human studies as NCEs transition from the preclinical stage to clinical development as the Investigational New Drug (IND) application is cleared? Indeed, the excitement partly stems from the fact that bioanalysis holds the key to unlocking the first results of human pharmacokinetics using concentration data derived from the newly developed and validated assay. In our own little world, highlighted by whispers in the laboratory corridors about the novel assay, brain-storming sessions in the cubby-holes, a sense of 'imagination' that the entire company is watching and mere anticipation of a spectacular outcome makes it all a momentous occasion.

Playing the dual role of bioanalyst and pharmacokineticist in the beginning of his career, the author can relate to the fact that human assay development and bioanalysis of clinical samples is considered to be the key milestone of achievement for many budding scientists in the world of bioanalysis - associated with pride, recognition and, above all, the establishment of the nexus for obtaining elusive human pharmacokinetic data. Bioanalytical scenarios, in an industry setting and in terms of perceived importance, start from in vitro sample analysis, preclinical sample analysis and culminate in human bioanalysis. An analogy could be drawn between the various levels of education, starting from a bachelor's degree stepping up to master's degree and finally culminating in a doctoral dissertation. However, compared with yesteryears, today's bioanalytical scenario has evolved greatly, with the development of a range of analytical tools, laboratory automation, a great leap in infrastructure development aided with bioinformatics, well-defined protocols for every bioanalytical aspect including quality-scrutiny systems, a repository of know-hows readily available and, above all, exceptional technical advancement, which in totality have enabled bioanalysts to accomplish things with speed and finesse.

Regardless of the assay type employed and the present-day nuances, questions still exist in the mind of bioanalysts as they get ready to take the challenge of developing the first assay for human samples. Most notably, these questions include:

- What should the sensitivity of the assay be?

- What should the range for establishing calibration curves be?

- Is there a need for the dilution of samples and, if so, should the dilution factor be five-fold or 20-fold?

- What is already known about this class of compound(s) and how can it be used to guide the bioanalysis in question?

- What are the key learnings from preclinical data and how could they be used to develop and/or fine tune the development of the human assay?

Although it is a personal and professional achievement to develop an assay to a single digit $\mathrm{pg} / \mathrm{ml}$ sensitivity level (or perhaps even lower), the key question to ask would be 'is there a need for this'? What would this serve? Should one be wasting resources and efforts in achieving a dream sensitivity that may never find any use as the compound progresses in higher doseescalation phases in the first-in-human study? The other side of the debate should perhaps focus on asking the question of 'what would be the limitation in not achieving rock-bottom sensitivity? An easy, rather simple answer to that would be that it may result in a lack of proper characterization of the disposition profile of the NCE in question at the first dose level in humans. Since this dose level merely serves as the safest dose to begin human



Nuggehally R Srinivas Suramus Biopharm., JP Nagar I Phase, Bangalore, 560078 , India

Tel.: +9l 9902977255

E-mail: srini.suramus@ yahoo.com 
evaluation of the compound and, perhaps, may never be tested again, would we care to know the intricacies of the pharmacokinetic disposition at this dose level? Through the author's own experience in countless drug-development programs, the lower quartile of the calibration curves developed for the first assay(s) in humans will never find utility as the compound advances in development. In fact, on many occassions, consideration has been given to reducing the sensitivity and narrowing the calibration curve ranges to manageable levels (i.e., 25-100-fold levels) rather than outlandish 1000-fold levels.

\section{"What are the key learnings from preclinical data and how could they be used to develop and/or fine tune the development of the human assay?"}

By the same notion, a bioanalyst should not become carried away with stretching the upper boundary of the calibration curve, however tempting the opportunity may appear. No doubt this may be extremely beneficial in first-inhuman studies since higher drug concentrations are likely to be manifested as the dose escalation may reach, by intentional design, severalfold higher than the expected and/or anticipated therapeutic dose. Would it not be prudent to fix the top end of the calibration curve to reflect a satisfactory and manageable range and, by doing so, ensure that validation of dilution samples is properly carried out to offer flexibility in the analysis? It is the opinion of the author that even if $30 \%$ of the samples need to be diluted at the top dose level, it should not justify the extension of the upper calibration point.

While practical and pragmatic approaches need to be considered, it is always important to pay attention to the details during bioanalysis. For example, it should be anticipated that a compound undergoing first-pass metabolism (and/ or p-glycoprotein transport) is expected to show extremely low levels in the initial dose-escalation steps; however, the trend will rapidly reverse, as the dose level threshold reaches the saturation point. Therefore, the calibration curve ranges should factor for these scenarios proactively, as opposed to implementing assay modifications as a result of the first sample analyses. Another common concern that is typically flagged during the intial discovery/preclinical stages would be the preliminary metabolic pathways. Is the NCE a substrate for the polymorphic cytochrome P450 2D6 isozyme? For some reason, if this is not picked up from the initial in vitro metabolism data, it may pose immense problems in the bioanalysis of samples, as poor metabolizers for this isozyme may manifest significantly higher concentrations of the parent NCE. However, such situations can easily be countered by having a cushion of at least a 50 - or 100-fold spread in the calibration curve range.

The excitement factor would certainly increase if a parent compound needed to be monitored with Phase I and/or Phase II metabolites, in spite of the incredible method development and validation challenges it may throw at the bioanalyst. Adding to this complexity, there may be situations where analyses have to be performed for one or more metabolites without the use of an appropriate reference standard(s). The issue of chirality and/or the likelihood of stereoselective metabolism may add another bioanalytical dimension. In other cases, unplanned work may have to be performed to address unknown metabolite(s) and/or determine the likely reason(s) for explaining the unanticipated low exposure in humans. Therefore, a bioanalyst will be kept on his/her toes as the first-in-human bioanalytical work is in progress.

Overall, first-in-human assays offer a lot to bioanalysts, including learning, exploration of speculative concepts, anticipation, success in navigating through problems and, importantly, self belief, which all contribute to tremendous excitement. We have learnt through our experiences that a timely introspection may be a prudent way to get back to the original baseline ensuring that the noise levels are flushed out without causing interference for the next peaking phase.

\section{Financial \& competing interests disclosure}

The author has no relevant affiliations or financial involvement with any organization or entity with a financial interest in or financial conflict with the subject matter or materials discussed in the manuscript. This includes employment, consultancies, honoraria, stock ownership or options, expert testimony, grants or patents received or pending, or royalties.

No writing assistance was utilized in the production of this manuscript. 